\title{
Highly efficient flow-through catalytic reduction of methylene blue using silver nanoparticles functionalized cotton
}

\author{
Longbin $\mathrm{Qi}^{\mathrm{a}, \mathrm{d}}$, Keming Zhang ${ }^{\mathrm{c}}$, Wei Qin ${ }^{\mathrm{a}}$, Yunxia $\mathrm{Hu}^{\mathrm{b}, *}$ \\ ${ }^{\text {a }}$ CAS Key Laboratory of Coastal Environmental Processes and Ecological Remediation, Yantai Institute of Coastal Zone Research (YIC), Chinese Academy of Sciences \\ (CAS), Shandong Key Laboratory of Coastal Environmental Processes, YIC, CAS, Yantai, Shandong 264003, PR China \\ ${ }^{\mathrm{b}}$ State Key Laboratory of Separation Membranes and Membrane Processes, School of Materials Science and Engineering, Tianjin Polytechnic University, Tianjin 300387, \\ PR China \\ ${ }^{\mathrm{c}}$ School of Environmental Science and Engineering, Tianjin Polytechnic University, Tianjin 300387, PR China \\ ${ }^{\mathrm{d}}$ University of Chinese Academy of Sciences, Beijing 100049, PR China
}

\section{H I G H L I G H T S}

- Ag NPs were immobilized on cotton via a one-step method.

- The Ag NPs modified cotton was used for flow-through catalytic reduction of methylene blue.

- Under flow-through mode, the permeation flux at $2000 \mathrm{LMH}$ with $>$ $99 \%$ reduction efficiency was obtained.

- The Ag NPs on cotton were stable and released very slowly.

\section{A R T I C L E I N F O}

\section{Keywords:}

Flow through reactor

Silver nanoparticles

Catalysis

Dye degradation

\section{G R A P H I C A L A B S T R A C T}

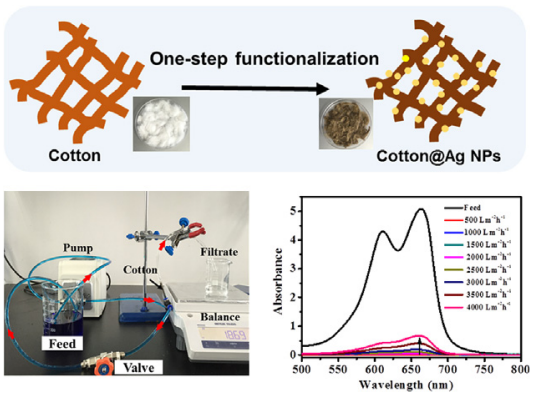

\begin{abstract}
A B S T R A C T
Organic dye pollution has become a serious problem threating environment and human health. Noble metal nanoparticles based catalytic degradation of organic dyes has attracted intensive attentions due to their outstanding catalytic activities. Compared with the catalytic systems using free noble metal nanoparticles, the flowthrough systems based on the immobilized noble metal nanoparticles are more suitable for both high efficiency reaction and recycling of nanoparticles. In this study, the fibrous natural material cotton is selected as the support of silver nanoparticles (Ag NPs) and used for flow-through catalytic reduction of methylene blue (MB). $\mathrm{Ag}$ NPs were one-step immobilized onto cotton by immersing the cotton into a mixture solution of silver nitrate, poly (ethylene glycol) methyl ether thiol and dopamine. The reaction rate constant $\mathrm{K}$ of $\mathrm{Ag}$ NPs functionalized cotton towards MB reached high up to $0.478 \mathrm{~min}^{-1}$. The flow-through system with Ag NPs functionalized cotton was operated to achieve a super high permeation flux of $2000 \mathrm{~L} \mathrm{~m}^{-2} \mathrm{~h}^{-1}$ and also a very high MB reduction of more than 99\%, which is far better than the reported results (below $500 \mathrm{~L} \mathrm{~m}^{-2} \mathrm{~h}^{-1}$ ). Moreover, the Ag NPs on cotton were very stable and released very slowly. After $24 \mathrm{~h}$ flowing-through test, only $0.9 \%$ of silver lose from the cotton. Our work provides a facile and applicable way to prepare the low-cost catalysts with high performances for continuous and efficient dye-containing waste water treatment.
\end{abstract}

\footnotetext{
* Corresponding author.

E-mail address: yunxiahu@tjpu.edu.cn (Y. Hu).
} 


\section{Introduction}

Organic dyes in wastewater have become a serious problem threating environment and human health [1-3]. Up to date, numerous technologies have been developed to remove organic dyes from waste water, such as physical absorption [1,4], membrane filtration $[5,6]$, chemical degradation [7] and biodegradation [8]. Among them, catalytic degradation of organic dyes using noble metal nanoparticles has attracted intensive attentions due to their outstanding catalytic activities and efficient color removal. However, there are several challenges limiting the practical use of these nanoparticle catalysts. For example, it is difficult to recycle these colloidal nanoparticles in aqueous solution for sustainable use [9]. Besides, the nanoparticles tend to aggregate because of their large surface area and high surface energy, which have strong negative effects on their chemical catalytic properties and stability [10].

Immobilization of noble metal nanoparticles on a support is considered as an effective approach to solve these above problems [11]. Nowadays, various materials have been employed as supports such as grapheme [12], $\mathrm{Fe}_{3} \mathrm{O}_{4}$ microspheres [13], zeolites [14], polymeric microsphere [15], etc. These supports not only contribute to the dispersion and stabilization of nanoparticles but also improve the reusability of noble metal nanoparticles. However, these catalysts still need to be separated from the mixture solution for reuse after the catalytic reaction. Coupling the catalytic reaction and catalyst separation together, porous filtration membranes have been used as such a support of noble metal nanoparticles to perform flow-through catalytic reaction for the removal of dyes in aqueous solutions [16]. For example, Subair et al. have synthesized gold nanoparticles on the microfiltration membrane for continuous flow-through catalytic degradation of organic dyes, which exhibited sustained catalytic activities and maintained more than 99\% degradation efficiency after 11 cycles [17]. The membrane matrix provides numerous pores to load large amount of nanoparticles ensuring high catalytic efficiency. Unfortunately, there is a trade-off between the flux and catalytic efficiency due to a short residence time of a dye-containing solution in a filtration membrane. Generally, the membrane flux needs be maintained at a relatively low value to ensure the complete reduction of dyes (lower than $500 \mathrm{~L} \mathrm{~m}^{-2} \mathrm{~h}^{-1}$ to maintain 99\% degradation efficiency) [17-19]. Thus, the system efficiency of catalytic reduction is limited. It is desirable to further improve the flux of the flow-through catalytic system while maintaining nearly $100 \%$ reduction of dyes. Fibrous materials have a great potential to outperform better than porous filtration membranes when they are used as supports in the flow-through catalytic system due to the high specific surface area, high loading capacity of catalysts and high permeability $[7,20,21]$. Fibrous cellulose materials, such as cotton, have unique features of good chemical stability, high porosity and good flexibility, and can be a good choice as the candidate support.

Immobilization of nanoparticles on a filtration support offers interesting possibilities for the flow-through catalysis reaction. It is worth noting that the nanoparticles leaching from support can significantly reduce the performance of catalysis [7]. To firmly bind the nanoparticles to the support, the surface of support is engineered using the anchors of nanoparticles such as amino groups [22], thiol groups [23], polyelectrolytes [24], etc. However, these methods are complicated and the loading of nanoparticles is very low. Recently, inspired by the mussel bio-adhesion strategy, several research groups have successfully synthesized noble metal nanoparticles on supports via the first polydopamine deposition and the following nanoparticles generation to achieve the flow-through catalytic reduction of organic dyes $[7,18]$. However, this method still requires two steps and complex procedures. In order to improve the simplicity and universality of the nanoparticles functionalization onto the supports, we developed a one-step facile method to synthesize and immobilize of silver nanoparticles (Ag NPs) onto diverse supports [25]. The loading amount of Ag NPs was higher than most of the reported results. Besides, even under the condition of water flushing, the Ag NPs were still tightly bound to the support due to the adhesive nature of polydopamine.

In this study, the fibrous natural material cotton is selected as the support of the noble metal nanoparticles and used for the flow-through catalytic reduction of organic dyes. Ag NPs were one-step immobilized on cotton by immersing the cotton into a mixture solution of silver nitrate, poly (ethylene glycol) methyl ether thiol (PEG-SH) and dopamine. The methylene blue (MB) was chosen as a model organic dye. The performances for the catalytic reduction of MB using the catalyst of Ag NPs loaded cotton (cotton@Ag NPs) were systematically investigated under the continuous flow condition using $\mathrm{MB}$ solution containing $\mathrm{NaBH}_{4}$ as a feed solution. Moreover, the reusability of cotton@Ag NPs was also investigated, and the silver leaching from the cotton@Ag NPs was measured during the flow-through catalytic reaction. Our work aims to develop a simple and applicable way to prepare the low-cost catalyst with high performances for continuous and efficient dye-containing waste water treatment.

\section{Experimental section}

\subsection{Materials}

Poly (ethylene glycol) methyl ether thiol (PEG-SH, $\mathrm{M}_{\mathrm{n}}: 800 \mathrm{~g} / \mathrm{mol}$ ), dopamine and sodium borohydride $\left(\mathrm{NaBH}_{4}\right)$ were purchased from Sigma Aldrich (St. Louis, MO, USA). Degreasing cotton and silver nitrate were received from Sinopharm Chemical Reagent Beijing Co., Ltd, China. Methylene blue (MB) was obtained from Dengke Chemical Reagent Co., Ltd China. All chemical regents were used without further purification.

\subsection{Preparation of Ag NPs functionalized cotton}

The Ag NPs functionalized cotton was prepared following our previous work [25]. Briefly, $1 \mathrm{~g}$ degreasing cotton was immersed in $100 \mathrm{~mL}$ aqueous solution containing $0.1 \mathrm{mM}$ PEG-SH and $2 \mathrm{mM}$ silver nitrate for $10 \mathrm{~min}$. Then, $200 \mathrm{mg}$ dopamine was dissolved in the above mixture solution and then in situ reduction and immobilization of Ag NPs on cotton occurred at room temperature under magnetic stirring for $4 \mathrm{~h}$. After that, the cotton was thoroughly rinsed with deionized water and dried in an oven.

\subsection{Characterizations}

UV-visible spectra of the MB solution were collected at a wavelength from $500 \mathrm{~nm}$ to $800 \mathrm{~nm}$ using a UV-Vis spectrophotometer (UV2700, Shimadzu, Japan). Morphology of cotton@Ag NPs was observed using a scanning electron microscope (SEM, S-4800, Hitachi, Japan). Each sample was vacuum dried, sputter-coated with platinum before SEM observation. The elemental compositions of cotton@Ag NPs were analyzed by an energy dispersive X-ray microanalyzer (EDX, EX-350, Horiba, Japan) under a voltage of $15 \mathrm{keV}$. The content of Ag NPs was measured using a thermo gravimetric analyzer (TGA, Mettler Toledo, Switzerland) at a temperature range from $50{ }^{\circ} \mathrm{C}$ to $800{ }^{\circ} \mathrm{C}$ in air. The concentration of silver ions in solution was analyzed by an inductively coupled plasma mass spectrometry (ICP-MS, ELAN DRC II, PerkinElmer (Hong Kong) Ltd.). Before ICP-MS analysis, the solution was acidified with 3.5\% nitric acid. The size distribution of Ag NPs in solution was measured using a Zetasizer Nano ZS dynamic light scattering (DLS) system (ZS90, 96Malvern, UK).

\subsection{Catalytic reduction of $M B$ using cotton@Ag NPs}

The catalytic reduction experiment was performed to evaluate the catalytic activities of cotton@Ag NPs following the reported work [26]. $50 \mathrm{mg}$ cotton@Ag NPs was immersed into $10 \mathrm{~mL} 50 \mathrm{mg} \mathrm{L}^{-1} \mathrm{MB}$ solution in the presence of $0.02 \mathrm{M} \mathrm{NaBH}_{4}$ under continuous shaking. At a 
certain time, the mixture solution was taken out to collect its UV-Vis spectra at the wavelength from $500 \mathrm{~nm}$ to $800 \mathrm{~nm}$. As a control, the same procedures were performed using the pristine cotton. The reusability of cotton@Ag NPs was tested under the same condition. After each test, cotton@Ag NPs was collected through 5 min centrifugation at $2000 \mathrm{rpm}$ to remove the aqueous solution and then re-immersed into $10 \mathrm{~mL}$ fresh MB solution in the presence of $\mathrm{NaBH}_{4}$ for continuous catalytic reduction. The concentration of MB was determined by measuring the UV-Vis absorbance of the mixture solution at $665 \mathrm{~nm}$. The reduction efficiency of $\mathrm{MB}$ was calculated following the equation:

$\mathrm{R}_{\mathrm{MB}}=\left(\frac{\mathrm{C}_{0}-\mathrm{C}_{\mathrm{t}}}{\mathrm{C}_{0}}\right) \times 100 \%$

where $R_{M B}$ is the reduction efficiency of $M B, C_{0}\left(\mathrm{mg} \mathrm{L}^{-1}\right)$ and $C_{t}$ $\left(\mathrm{mg} \mathrm{L}^{-1}\right.$ ) are the initial MB concentration and the real-time MB concentration, respectively.

\subsection{Flow-through catalytic reduction of $M B$ using cotton@Ag NPs}

For a flow-through catalytic reduction experiment, $50 \mathrm{mg}$ cotton@Ag NPs was tightly crammed into a polyvinyl chloride tube with a diameter of $6 \mathrm{~mm}$ and an effective length of $20 \mathrm{~mm}$ as shown in Fig. S1. The tube was installed in the continuous filtration system. The MB solution $\left(50 \mathrm{mg} \mathrm{L}^{-1}\right)$ containing $\mathrm{NaBH}_{4}(0.02 \mathrm{M})$ was used as the feed solution. For each test, the flow-through flux was adjusted to a determined value. Then, $20 \mathrm{~g}$ of the MB solution after flowing through the cotton@Ag NPs was taken to measure its UV-Vis absorbance at the wavelength from $500 \mathrm{~nm}$ to $800 \mathrm{~nm}$. The reduction efficiency of MB was calculated following the Eq. (1).

\section{Results and discussion}

\subsection{One-step synthesis and immobilization of Ag NPs on cotton}

The Ag NPs were one-step synthesized and immobilized on cotton via immersing the cotton into a mixture solution of silver nitrate, PEG$\mathrm{SH}$ and dopamine. During this process, dopamine served as a reducing agent and PEG-SH as a stabilizing agent. In the absence of PEG-SH, silver ions were reduced by dopamine, followed by precipitation immediately. This is due to the strong reducing capacity of dopamine and the strong oxidizing ability of silver ions. With the increasing of PEG-SH concentration from $0.1 \mathrm{mM}$ to $10 \mathrm{mM}$, the size of Ag NPs in the bulk solution decreased from $216.7 \mathrm{~nm}$ to $11.7 \mathrm{~nm}$ (Fig. S2). The PEG-SH could chelate silver ions via the thiol group, which slowed down the reduction process to synthesize Ag NPs in a controllable manner [25]. The influences of dopamine concentration and $\mathrm{AgNO}_{3}$ concentration on controlling the size of $\mathrm{Ag}$ NPs were further investigated and results were shown in Table S1. With the increasing of dopamine concentration from $0.2 \mathrm{mg} \mathrm{mL}^{-1}$ to $20 \mathrm{mg} \mathrm{mL}^{-1}$, the size of Ag NPs in the bulk solution increased from $30.1 \mathrm{~nm}$ to $311.4 \mathrm{~nm}$. Similarly, with the increasing of $\mathrm{AgNO}_{3}$ concentration from $0.2 \mathrm{mM}$ to $200 \mathrm{mM}$, the size of Ag NPs in the bulk solution increased from $23.1 \mathrm{~nm}$ to $284.1 \mathrm{~nm}$. The low concentration of precursors may result in a long modification time. While, the high concentration of precursors may result in an uncontrollable reaction and synthesis of large particles. Therefore, the condition ( $1 \mathrm{mM}$ PEG-SH, $2 \mathrm{mg} \mathrm{mL}^{-1}$ DOPA and $20 \mathrm{mM} \mathrm{AgNO}_{3}$ ) was selected to synthesize Ag NPs in the range of nanometer size.

After Ag NPs functionalization, the color of cotton turned from white to brown as shown in Fig. 1A and D. The surface morphology of cotton and cotton@Ag NPs were further characterized using SEM. As shown in Fig. 1B and E, both cotton and cotton@Ag NPs show a fibrous structure consisting of fiber with an average diameter about $15 \mu \mathrm{m}$ and nanoparticles with an average diameter about $20 \mathrm{~nm}$. From the high magnification SEM images shown in Fig. 1C and F, the pristine cotton fibers have a smooth surface. After Ag NPs functionalization, numerous bright nanoparticles were distributed on the surface of cotton fibers. In addition, the signal of silver element was found in the EDX spectrum of cotton@Ag NPs as shown in Fig. S3, confirming the successful immobilization of Ag NPs on cotton. To investigate the oxidative state of Ag NPs, XPS was employed to identify the state of silver on the substrate. As shown in Fig. S4, two different species of $\mathrm{Ag} 3 \mathrm{~d}_{5 / 2}$ were observed, and silver $\mathrm{Ag}^{0}$ was dominantly found at $368.1 \mathrm{eV}$ and $\mathrm{Ag}^{+}$was clearly detected at $368.7 \mathrm{eV}$. The silver content in the cotton@Ag NPs was quantified using TGA, which was calculated to be $3.58 \%$ by subtracting the residual mass of pristine cotton from the residual mass of cotton@Ag NPs, as shown in Fig. S5.

The permeate performance of cotton was investigated with increasing the applied pressure and found that there was no obvious change of flux before and after the loading of Ag NPs onto cotton, as shown in Fig. S6. Additionally, the water flux of pristine cotton and Ag NPs loaded cotton increased linearly with the applied pressure in the range of $0.1-0.3 \mathrm{bar}$, since the increased driving force induced fast water transport. The pure water permeability coefficients of pristine cotton and cotton@Ag NPs were measured to be 34,129.2 \pm 773.9 $\mathrm{LMH} \mathrm{bar}^{-1}$ and 33,431.1 \pm 1957.2 LMH bar ${ }^{-1}$, respectively, which is much higher than that of ultrafiltration and microfiltration membranes.

\subsection{The catalytic properties of cotton@Ag NPs}

The catalytic properties of cotton@Ag NPs for MB were investigated in the presence of $\mathrm{NaBH}_{4}$. The reaction was monitored by measuring the color fading of MB solution using a UV-Vis spectrophotometer at the maximum absorbance wavelength $(\lambda=665 \mathrm{~nm})$. As shown in Fig. 2A, the absorbance intensity of MB solution at $665 \mathrm{~nm}$ decreased rapidly with prolonging the reaction time, indicating the gradual catalytic reduction of MB. During this reaction, the MB molecule accepts electron from $\mathrm{BH}_{4}{ }^{-}$to form colorless leucomethylene blue, which occurs on the surface of Ag NPs [27]. The role of the Ag NPs is to facilitate the electron transfer from electron donor to electron acceptor [28]. For the control experiments using the pristine cotton, the absorbance intensity of MB solution decreased slightly as shown in Fig. 2B, which is attributed to the adsorption of cotton.

Fig. 2C shows the linear relationship between $\ln \left(\mathrm{C}_{t} / \mathrm{C}_{0}\right)$ and the reaction time, confirming the pseudo first-order kinetics of the MB solution color removal when using the pristine cotton and cotton@Ag NPs. The slope of the line in Fig. 2C shows the catalytic rate constant K. The larger the absolute value of $\mathrm{K}$ is, the faster the catalytic reaction rate is. The K value for MB catalytic reduction using cotton@Ag NPs was calculated to be $0.478 \mathrm{~min}^{-1}$. While, the $\mathrm{K}$ value for pristine cotton was only $0.014 \mathrm{~min}^{-1}$, which is mainly due to the absorption of $\mathrm{MB}$ in pristine cotton without the catalytic reaction involved. Moreover, the loading amount of Ag NPs on support is an important factor affecting the catalytic rate [18]. Our previous study showed that the loading amount of Ag NPs loading on support increased with prolonging the immersion time of the support in the $\mathrm{AgNO}_{3} / \mathrm{mPEG}$-SH/DOPA solution [25]. Fig. S7 shows that both the number of Ag NPs and the size of Ag NPs increased significantly. With prolonging the reaction time solution from $1 \mathrm{~h}$ to $12 \mathrm{~h}$, the diameter of Ag NPs increased from $15.2 \pm 3.2 \mathrm{~nm}$ to $42.4 \pm 10.3 \mathrm{~nm}$. Fig. $2 \mathrm{D}$ shows that the $\mathrm{K}$ value increased from $0.024 \mathrm{~min}^{-1}$ to $0.478 \mathrm{~min}^{-1}$ with prolonging the immersion time of cotton in the $\mathrm{AgNO}_{3} / \mathrm{mPEG}$-SH/DOPA solution from $1 \mathrm{~h}$ to $4 \mathrm{~h}$, which is due to the increased loading of Ag NPs on cotton providing more available surface areas for catalytic reaction. While, the $\mathrm{K}$ value reached to a plateau with further increasing the immersion time from $4 \mathrm{~h}$ to $12 \mathrm{~h}$, which might be attributed to the lack of $\mathrm{NaBH}_{4}$ to reduce MB [26].

The flow-through catalytic performance of the cotton@Ag NPs catalyst was conducted in a continuous filtration system as shown in Fig. S1. The flux of MB containing feed solution was varied from $500 \mathrm{~L} \mathrm{~m}^{-2} \mathrm{~h}^{-1}$ (LMH) to $4000 \mathrm{LMH}$, and the MB concentration in the 


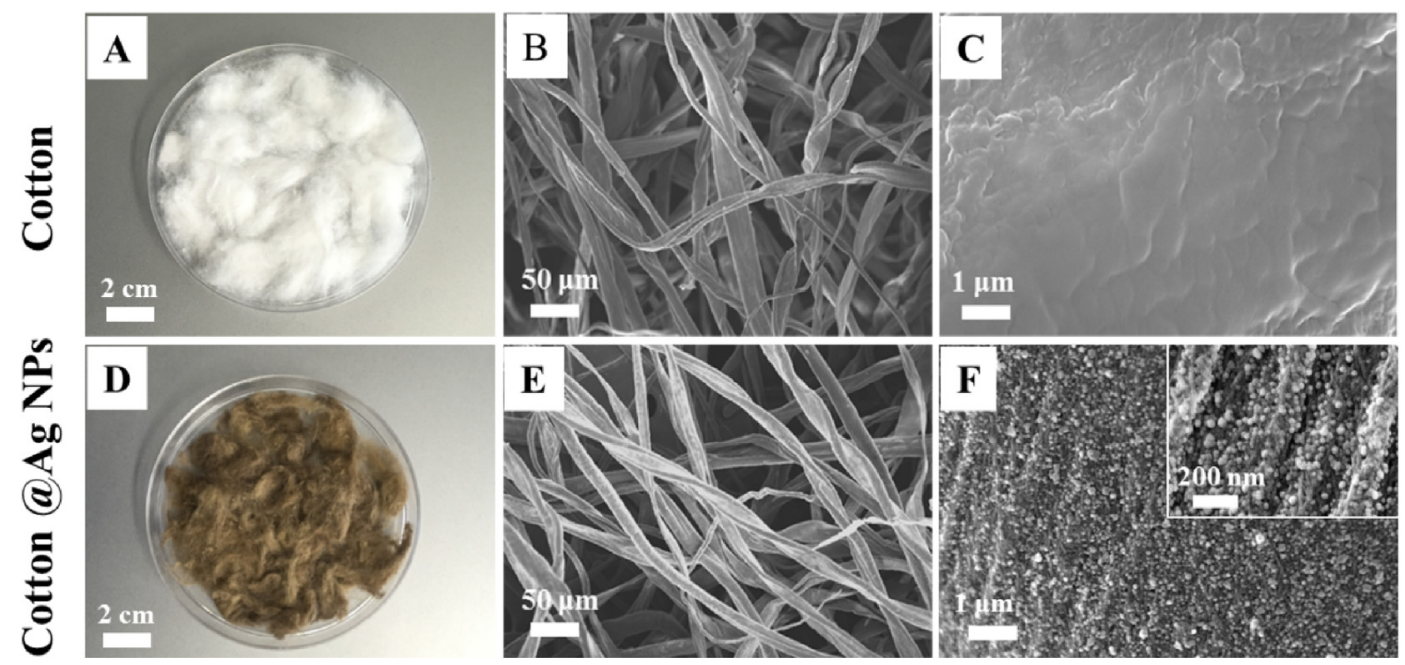

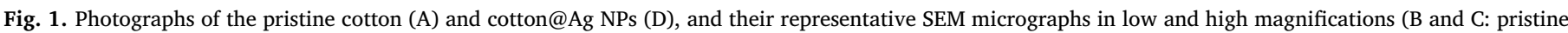
cotton, E and F: cotton@Ag NPs).
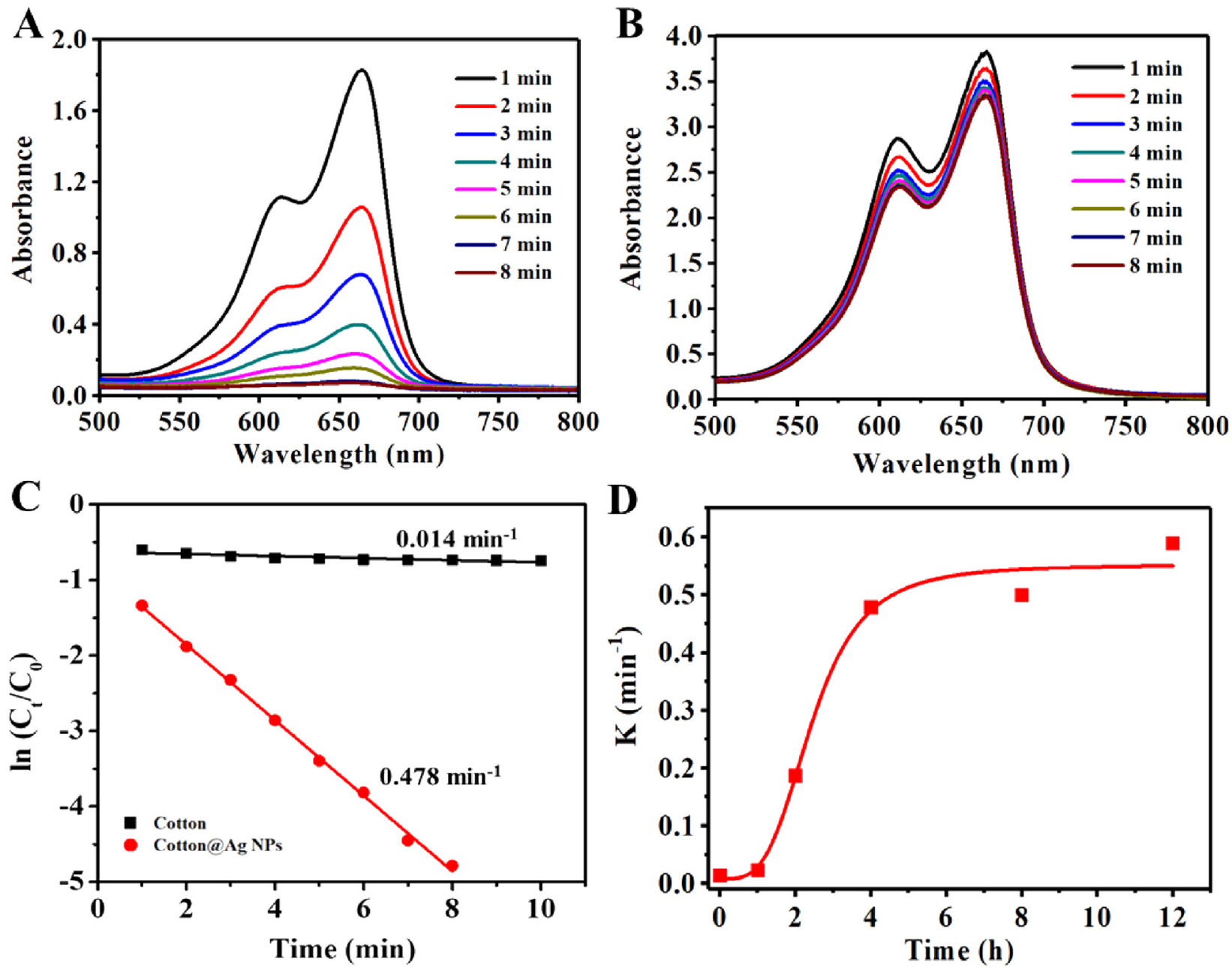

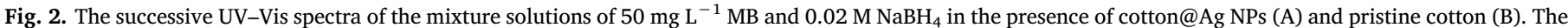

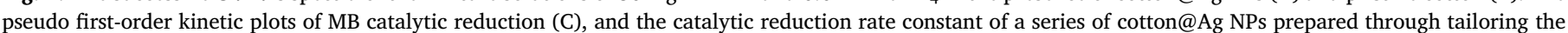

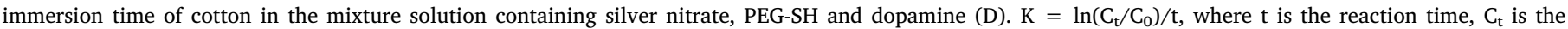
corresponding concentration of $\mathrm{MB}$ at the reaction time of $\mathrm{t}$, and $\mathrm{C}_{0}$ is the initial concentration of $\mathrm{MB}$. 
A

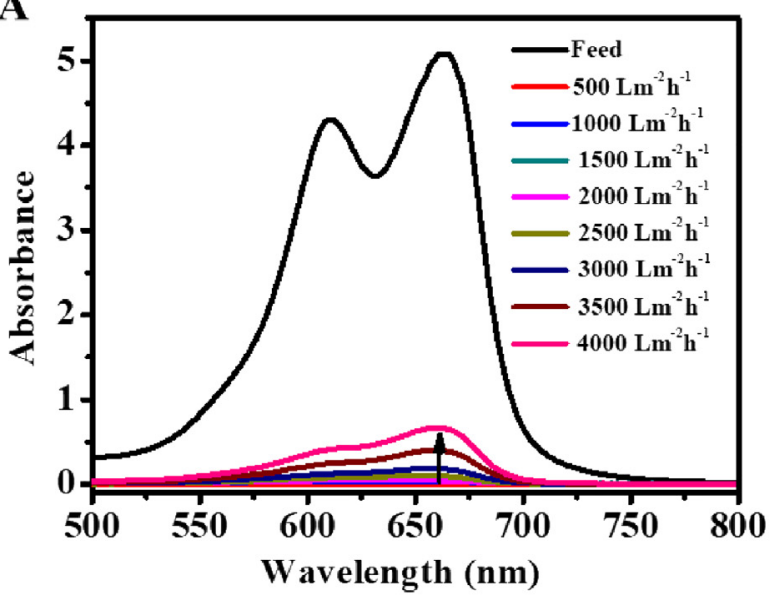

B

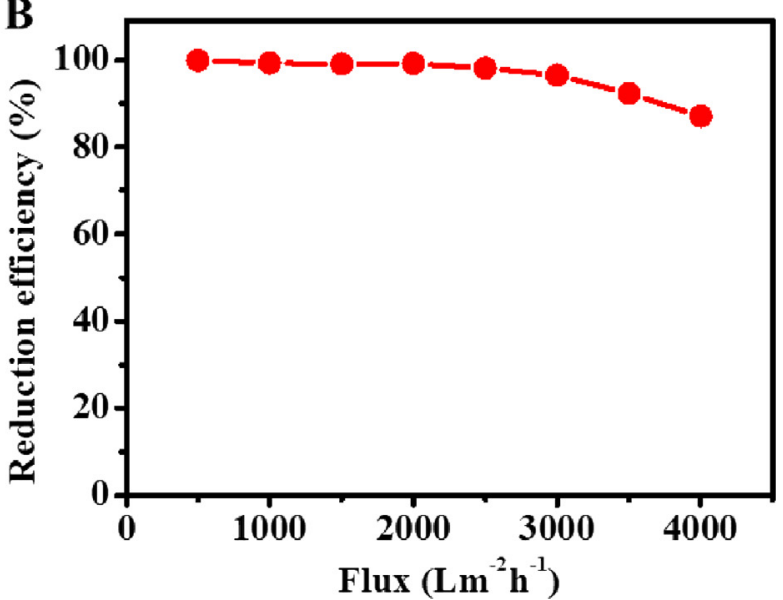

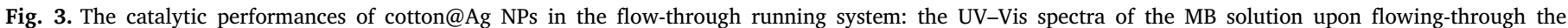

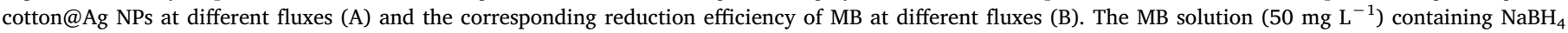
$(0.02 \mathrm{M})$ was used as the feed solution.

filtrate was monitored by a UV-Vis spectrophotometer. As shown in Fig. 3, the absorbance peak of MB in the filtrate at $665 \mathrm{~nm}$ disappeared and the MB was completely reduced after the feed solution of MB flowed through the catalyst of cotton@Ag NPs at the flux of lower than $2000 \mathrm{LMH}$. The absorbance peak of MB in the filtrate at $665 \mathrm{~nm}$ started to be detected when the flux was higher than $2000 \mathrm{LMH}$, and the concentration of MB in the filtrate increased when the flux was higher. It is worth noting that under the flow-through conditions with rapid convective mass transport of reactants to the immobilized Ag NPs, the reduction efficiency of MB depends on the kinetics or mass flow, rather than diffusion. With the increasing of the flux from 500 LMH to $2000 \mathrm{LMH}$, the reduction efficiency of MB maintained to be higher than 99\%. The reduction efficiency of MB declined from $98.1 \%$ to $87.1 \%$ with further increasing the flux from $2500 \mathrm{LMH}$ to $4000 \mathrm{LMH}$, which is mainly due to the decreased residence time of MB solution with the cotton@Ag NPs catalyst. The degradation of dyes at the high flux is of great significance for the practical application in environmental remediation. The concentration of $\mathrm{MB}$ and $\mathrm{NaBH}_{4}$ are two important factors affecting the degradation efficiency. The concentration of MB and $\mathrm{NaBH}_{4}$ can also be optimized to achieve the better performance of catalyst $[17,26]$.

Compared with the performances of the reported flow-through catalytic systems, our cotton@Ag NPs catalyst based system exhibited a distinctive high flux (2000 LMH) while maintaining a super high reduction efficiency (>99\%). For example, Hu et al. prepared the $\mathrm{Ag}$ NPs-modified porous polypropylene membrane and achieved the best performance at the flux of about $210 \mathrm{LMH}$ when used for the flowthrough catalytic reduction of MB [19]. Subair et al. developed the gold nanoparticles incorporated poly (ethylene terephthalate) membrane for the catalytic degradation of organic dyes and obtained more than $98 \%$ degradation for MB at the flux of $473 \mathrm{LMH}$ [17]. Therefore, our catalytic flow system of cotton@Ag NPs performed far better than the reported ones, which is mainly because of a high loading capacity of $\mathrm{Ag}$ NPs and very loose fibrous structure of cotton having a high surface area.

\subsection{Sustainable catalytic performance and stability of cotton@Ag NPs}

To evaluate the sustainable catalytic performance of cotton@Ag NPs, the continuous flow-through catalytic reaction was performed under the flux of $2000 \mathrm{~L} \mathrm{~m}^{-2} \mathrm{~h}^{-1}$. The feed solution (MB $50 \mathrm{mg} \mathrm{mL}^{-2}$, $\mathrm{NaBH}_{4} 0.02 \mathrm{M}$ ) flowed through the cotton@Ag NPs and every $20 \mathrm{~mL}$ permeate solution was taken out to measure the concentration of MB and thus to calculate the reduction efficiency of MB. Fig. 4A observes a very high reduction efficiency of MB during the flow-through catalytic reaction and presents a very small decline of the MB reduction efficiency from $99.9 \%$ to $94.6 \%$ after $260 \mathrm{~mL}$ permeate was collected when $12.6 \mathrm{mg}$ MB was reduced. For the pristine cotton, although the reduction efficiency of $\mathrm{MB}$ reached to $60.9 \%$ during the first $20 \mathrm{~mL}$ of filtrate due to the MB adsorption in cotton, and then rapidly decreased to zero when the permeate volume reached to $40 \mathrm{~mL}$ and above, because of the absorption saturation of $\mathrm{MB}$ in cotton. These results confirm that the cotton@Ag NPs is capable of performing highly efficient and long-term catalytic reduction of $\mathrm{MB}$ in the flow-through condition.

The long-term catalytic performance of cotton@Ag NPs was also investigated in the static condition for many cycles. During one cycle of the test, the reduction efficiency of $\mathrm{MB}$ was measured upon incubating $50 \mathrm{mg}$ cotton@Ag NPs with the feed solution containing $10 \mathrm{~mL}$ MB solution $\left(50 \mathrm{mg} \mathrm{mL}{ }^{-1}\right.$ ) and $0.02 \mathrm{M} \mathrm{NaBH}_{4}$ for $10 \mathrm{~min}$. For the next cycle of the test, the solution in the system was emptied through centrifugation, and another $10 \mathrm{~mL}$ of fresh feed solution was filled to incubate with cotton@Ag NPs, the performance of cotton@Ag NPs was evaluated in terms of the MB reduction efficiency following the same protocol as above. Fig. 4B illustrates that the reduction efficiency of MB was stably high above $98 \%$ during the first 10 cycles when $4.9 \mathrm{mg}$ of MB was reduced, indicating the long-term high catalytic performance of cotton@Ag NPs and the good reusability of cotton@Ag NPs. The reduction efficiency of MB decreased gradually to $85.4 \%$ with further increasing the cycle number from 10 to 20 , suggesting a slow deterioration of catalytic properties of cotton@Ag NPs. The decreases of catalytic reduction efficiency in both flow-through mode and static condition may be attributed to the following two reasons: the loss of $\mathrm{Ag}$ NPs and the passivation of Ag NPs. It has been reported that the noble metal nanoparticles could be passivated during the catalytic reaction [29]. The binding of reaction product onto their surface may reduce their catalytic activities. Such phenomenon can be alleviated by a simple ethanol cleaning [19,30].

To investigate the long-term stability of cotton@Ag NPs, the flowthrough catalytic reaction was carried out at the flux of $2000 \mathrm{LMH}$ using the MB solution ( $50 \mathrm{mg} \mathrm{mL}^{-1}$ ) containing $0.02 \mathrm{M} \mathrm{NaBH}_{4}$ as the feed solution, and the concentration of silver ions in the filtrate was measured using ICP-MS. Fig. 5 shows the release profile of silver ions from cotton@Ag NPs during the flow-through catalytic reaction. At the initial stage of the experiment, the concentration of silver ions in the filtrate was $34.1 \mathrm{ppb}$ when the permeate volume was $50 \mathrm{~mL}$, and then decreased rapidly with the increase of permeate volume to $300 \mathrm{~mL}$, exhibiting a fast release behavior of silver from cotton@Ag NPs at the beginning of the flow-through operation. Then, the concentration of 

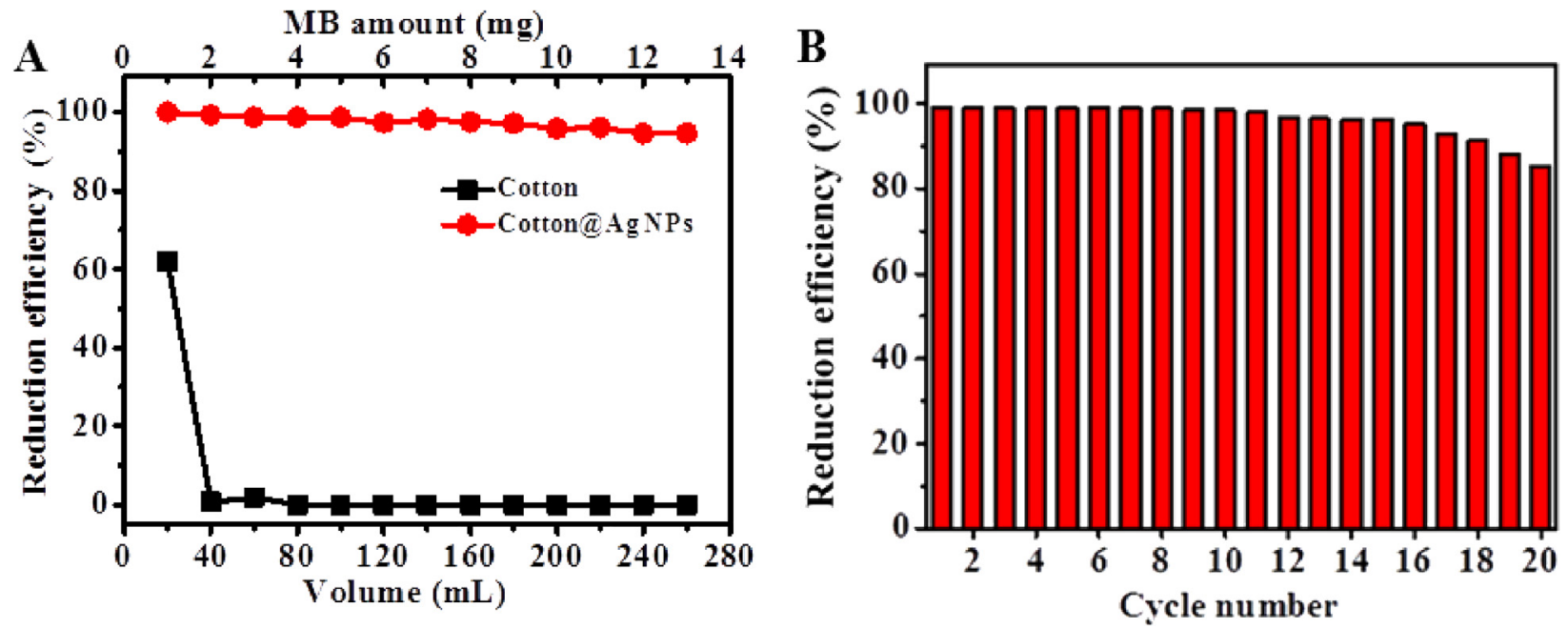

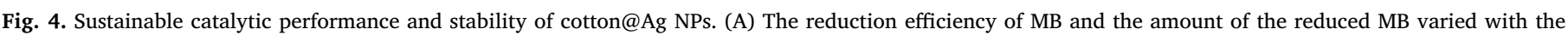

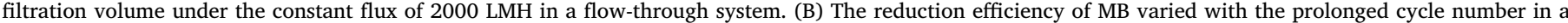
static condition. The feed solution in both of the flow-through system and static system: $5 \mathrm{mg} \mathrm{L^{-1 }}$ cotton@Ag NPs, $0.02 \mathrm{M} \mathrm{NaBH}_{4}$ and $50 \mathrm{mg} \mathrm{L}^{-1} \mathrm{MB}$

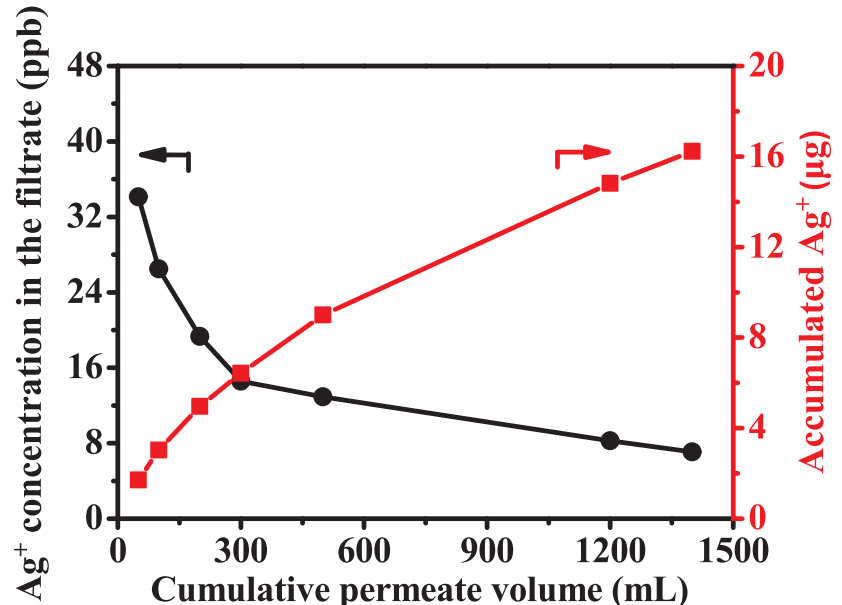

Fig. 5. The silver ions release profile from cotton@Ag NPs $(50 \mathrm{mg})$ during the flow-through catalytic reduction at the flux of $2000 \mathrm{~L} \mathrm{~m}^{-2} \mathrm{~h}^{-1}$. The MB solution $\left(50 \mathrm{mg} \mathrm{L}^{-1}\right)$ containing $\mathrm{NaBH}_{4}(0.02 \mathrm{M})$ was used as the feed solution.

silver ions in the filtrate slowly decreased to be $7.1 \mathrm{ppb}$ with increasing the permeate volume of $\mathrm{MB}$ solution to $1400 \mathrm{~mL}$, which are lower than the maximal contaminant limit of silver ions $(100 \mathrm{ppb})$ in drinking water [31]. Moreover, the amount of the accumulative silver ions after $24 \mathrm{~h}$ test was $16.2 \mu \mathrm{g}$, which was calculated to be only $0.9 \%$ of the total silver loading mass on cotton. This result suggests that about $99.1 \%$ of silver was still in the form of cotton@Ag NPs and the cotton@Ag NPs has a great potential to be long-term stable and active. To further investigate the silver leaching from cotton@Ag NPs, the morphologies of cotton@Ag NPs before and after 20-cycles test were observed using SEM. As shown in Fig. 6, a slight decrease in the number of Ag NPs is observed, which is responsible for the relative fast loss of Ag NPs during the initial test. Our previous study also shows that not only PEG-SH but also polydopamine were found on the surface of Ag NPs [25]. The Oand N-based groups in polydopamine could serve as anchors for Ag NPs, which improves the stability of Ag NPs. Therefore, we propose that the decrease of catalytic reduction efficiency is mainly due to the passivation of Ag NPs.

The stability of cotton@Ag NPs was also investigated by the treatment with acid and base solution. The cotton@Ag NPs was immersed in $0.01 \mathrm{M} \mathrm{HCl}$ or $0.01 \mathrm{M} \mathrm{NaOH}$ for $12 \mathrm{~h}$ and then washed with deionized water. During this process, no obvious structural collapse of cotton was observed (Fig. S8). The cotton@Ag NPs was treated in the abovementioned acid and base conditions for $12 \mathrm{~h}$ and then washed with deionized water thoroughly. The catalytic reduction experiments were performed to evaluate the catalytic activities of cotton@Ag NPs upon acid or base treatment. As shown in Fig. S9, the acid treated cotton@Ag NPs still exhibited excellent catalytic activities. The catalytic rate constant K of the acid treated cotton@Ag NPs was $0.453 \mathrm{~min}^{-1}$, which was slightly lower than the catalytic rate constant $\mathrm{K}\left(0.478 \mathrm{~min}^{-1}\right)$ of the untreated cotton@Ag NPs. While, the K value of the base treated cotton@Ag NPs decreased to $0.263 \mathrm{~min}^{-1}$. Generally, the Ag NPs was stable under base condition than under acidic condition. This unexcepted phenomenon is mainly due to the detachment of polydopamine coating from cotton and thus the loss of Ag NPs from cotton.
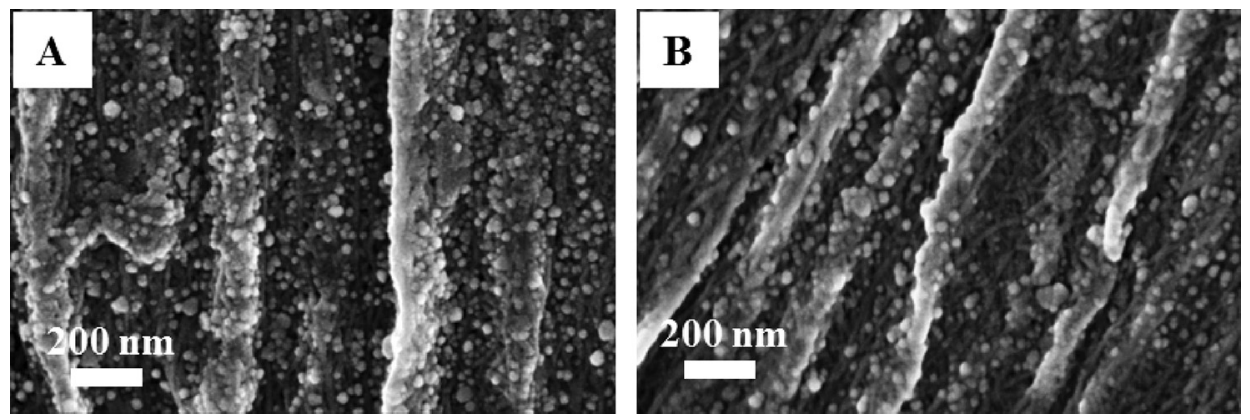

Fig. 6. SEM mages of the cotton@Ag NPs before (A) and after (B) 20-cycle tests. The experimental conditions: $50 \mathrm{mg}$ cotton@Ag NPs incubated with $0.02 \mathrm{M}$ NaBH 4 and $50 \mathrm{mg} \mathrm{L}^{-1} \mathrm{MB}$ in $10 \mathrm{~mL}$ aqueous solution for $10 \mathrm{~min}$ for each cycle. 
It has been reported that polydopamine was detached more easily and quickly in the alkaline condition than in acidic condition, and the detachment of polydopamine was faster than the dissolution of Ag NPs. [32-33].

\section{Conclusions}

In this study, Ag NPs was loaded on the low-cost natural support cotton through one step synthesis and immobilization of Ag NPs via the modified dopamine chemistry and then Ag NPs loaded cotton was used for the highly efficient flow-through catalytic reduction of MB. The synthesis and immobilization of Ag NPs on cotton was achieved by immersing the cotton into a mixture solution of silver nitrate, PEG-SH and dopamine and confirmed by the results of SEM and EDX. The MB solution (50 mg mL${ }^{-1}$ ) was completely decolorized within 8 min when using cotton@Ag NPs $(50 \mathrm{mg})$ as the catalyst and $\mathrm{NaBH}_{4}(0.02 \mathrm{M})$ as the reducing agent, and the reaction rate constant $\mathrm{K}$ reached high up to $0.478 \mathrm{~min}^{-1}$. Under the continuous flow condition, the cotton@Ag NPs exhibited highly efficient and sustainable catalytic performances at the high flux of feed solution filtrating through the catalyst. The reduction efficiency of MB maintained above $90 \%$ after $260 \mathrm{~mL}$ permeate solution was collected and tested at the flux of $2000 \mathrm{~L} \mathrm{~m}^{-2} \mathrm{~h}^{-1}$, which is far better than the reported results (below $500 \mathrm{~L} \mathrm{~m}^{-2} \mathrm{~h}^{-1}$ ). The cotton@Ag NPs could be reused for 20 cycles with only 14.6\% decline in the reduction efficiency of MB. Moreover, the Ag NPs on cotton had an excellent stability and released very slowly. $99.1 \%$ of silver was still in the form of cotton@Ag NPs after 24 h flowing-through test. Our work presents a highly efficient catalyst of cotton@Ag NPs with a great potential used in the flowing-through water treatment system for the color removal of dyes.

\section{Declaration of Competing Interest}

The authors declare that they have no known competing financial interests or personal relationships that could have appeared to influence the work reported in this paper.

\section{Acknowledgements}

The authors gratefully acknowledge the funding support from National Natural Science Foundation of China (No. 21476249, No. 51708408), Chang-jiang Scholars and Innovative Research Team in the University of Ministry of Education, China (No. IRT-17R80), Program for Innovative Research Team in University of Tianjin (No. TD13-5044) and the Science and Technology Plans of Tianjin (No. 18JCZDJC37100).

\section{Appendix A. Supplementary data}

Supplementary data to this article can be found online at https:// doi.org/10.1016/j.cej.2020.124252.

\section{References}

[1] I. Ali, New generation adsorbents for water treatment, Chem. Rev. 112 (2012) 5073-5091.

[2] K. Yu, S. Yang, C. Liu, H. Chen, H. Li, C. Sun, S.A. Boyd, Degradation of organic dyes via bismuth silver oxide initiated direct oxidation coupled with sodium bismuthate based visible light photocatalysis, Environ. Sci. Technol. 46 (2012) 7318-7326.

[3] C. Fernández, M.S. Larrechi, M.P. Callao, An analytical overview of processes for removing organic dyes from wastewater effluents, Trends. Anal. Chem. 29 (2010) $1202-1211$.

[4] W. Shi, G. Feng, H. Wang, C. Liu, Y. Fu, S. Yuan, H. Hui, L. Yang, Z. Kang, Carbon dots decorated magnetic $\mathrm{ZnFe}_{2} \mathrm{O}_{4}$ nanoparticles with enhanced adsorption capacity for the removal of dye from aqueous solution, Appl. Surf. Sci. 433 (2018) 790-797.
[5] Z. Shuang, W. Zhan, A loose nano-filtration membrane prepared by coating HPAN UF membrane with modified PEI for dye reuse and desalination, J. Membr. Sci. 524 (2017) 214-224.

[6] L. Qi, Y. Li, X. Ma, Q. Du, K. Sui, D. Wang, C. Wang, H. Li, Y. Xia, Filtration and adsorption properties of porous calcium alginate membrane for methylene blue removal from water, Chem. Eng. J. 316 (2017) 623-630.

[7] S. Lu, J. Yu, Y. Cheng, W. Qian, A. Barras, W. Xu, S. Szunerits, D. Cornu, R. Boukherroub, Preparation of silver nanoparticles/polydopamine functionalized polyacrylonitrile fiber paper and its catalytic activity for the reduction 4-nitrophenol, Appl. Surf. Sci. 411 (2017) 163-169.

[8] Y. Fu, T. Viraraghavan, Fungal decolorization of dye wastewaters: a review, Bioresour. Technol. 79 (2001) 251-262.

[9] X. Li, J.J. Lenhart, Aggregation and dissolution of silver nanoparticles in natural surface water, Environ. Sci. Technol. 46 (2012) 5378-5386.

[10] S. Gupta, P. Uhlmann, M. Agrawal, S. Chapuis, U. Oertel, M. Stamm, Immobilization of silver nanoparticles on responsive polymer brushes, Macromolecules 41 (2008) 2874-2879.

[11] M. Moritz, M. Geszkemoritz, The newest achievements in synthesis, immobilization and practical applications of antibacterial nanoparticles, Chem. Eng. J. 228 (2013) 596-613.

[12] Z. Zhe, X. Fugang, Y. Wenshu, G. Mingyi, W. Xiaodan, Z. Bailin, T. Jilin, A facile one-pot method to high-quality Ag-graphene composite nanosheets for efficient surface-enhanced Raman scattering, Chem. Commun. 47 (2011) 6440-6442.

[13] Y. Xie, B. Yan, H. Xu, J. Chen, Q. Liu, Y. Deng, H. Zeng, Highly regenerable musselinspired $\mathrm{Fe}_{3} \mathrm{O}_{4} @$ Polydopamine-Ag core-shell microspheres as catalyst and adsorbent for methylene blue removal, ACS Appl. Mater. Inter. 6 (2014) 8845-8852.

[14] Y. Xie, B. Yan, C. Tian, Y. Liu, Q. Liu, H. Zeng, Efficient removal of elemental mercury (Hg0) by SBA-15-Ag adsorbents, J. Mater. Chem. A 2 (2014) 17730-17734.

[15] B. Mao, O. An, Z. Xiao, B. Zhai, S. Zhai, Multifunctional hollow polydopaminebased composites $\left(\mathrm{Fe}_{3} \mathrm{O}_{4} / \mathrm{PDA} @ \mathrm{Ag}\right)$ for efficient degradation of organic dyes, RSC Adv. 6 (2016) 47761-47770.

[16] J. Wang, Z. Wu, T. Li, J. Ye, L. Shen, Z. She, F. Liu, Catalytic PVDF membrane for continuous reduction and separation of p-nitrophenol and methylene blue in emulsified oil solution, Chem. Eng. J. 334 (2018) 579-586.

[17] R. Subair, B.P. Tripathi, P. Formanek, F. Simon, M. Stamm, Polydopamine modified membranes with in situ synthesized gold nanoparticles for catalytic and environmental applications, Chem. Eng. J. 295 (2016) 358-369.

[18] G.H. Choi, D.K. Rhee, A.R. Park, M.J. Oh, S. Hong, J.J. Richardson, J. Guo, F. Caruso, P.J. Yoo, Ag nanoparticle/polydopamine-coated inverse opals as highly efficient catalytic membranes, ACS Appl. Mater. Inter. 8 (2016) 3250-3257.

[19] M.X. Hu, Q. Guo, J.N. Li, C.M. Huang, G. Ren, Reduction of methylene blue with Ag nanoparticle-modified microporous polypropylene membranes in a flow-through reactor, New J. Chem. 41 (2017) 6076-6082.

[20] R.S. Barhate, S. Ramakrishna, Nanofibrous filtering media: filtration problems and solutions from tiny materials, J. Membr. Sci. 296 (2007) 1-8.

[21] T. Niu, J. Xu, W. Xiao, J. Huang, Cellulose-based catalytic membranes fabricated by deposition of gold nanoparticles on natural cellulose nanofibres, RSC Adv. 4 (2014) 4901.

[22] L. Miao, Z. Guo, Y. Feng, R. Li, H. Pei, J. Zhang, J. Wang, Facile synthesis of silver nanoparticles on amino-modified cellulose paper and their catalytic properties, J. Mater. Sci. 53 (2018) 1568-1579.

[23] J. Yin, Y. Yu, Z. Hu, B. Deng, Attachment of silver nanoparticles (AgNPs) onto thinfilm composite (TFC) membranes through covalent bonding to reduce membrane biofouling, J. Membr. Sci. 441 (2013) 73-82.

[24] M. Fang, Z. Chen, S. Wang, H. Lu, The deposition of iron and silver nanoparticles in graphene-polyelectrolyte brushes, Nanotechnology 23 (2012) 085704.

[25] L. Qi, Z. Liu, W. Ning, Y. Hu, Facile and efficient in situ synthesis of silver nanoparticles on diverse filtration membrane surfaces for antimicrobial performance, Appl. Surf. Sci. 456 (2018) 95-103.

[26] N. Wang, Y. Hu, Z. Zhang, N. Wang, Y. Hu, Z. Zhang, Sustainable catalytic properties of silver nanoparticles supported montmorillonite for highly efficient recyclable reduction of methylene blue, Appl. Clay Sci. 150 (2017) 47-55.

[27] V.K. Vidhu, D. Philip, Catalytic degradation of organic dyes using biosynthesized silver nanoparticles, Micron 56 (2014) 54-62.

[28] M. Zhu, C. Wang, D. Meng, G. Diao, In situ synthesis of silver nanostructures on magnetic $\mathrm{Fe}_{3} \mathrm{O}_{4} @ \mathrm{C}$ core-shell nanocomposites and their application in catalytic reduction reactions, J. Mater. Chem. A 1 (2013) 2118-2125.

[29] C. Ray, S. Dutta, S. Sarkar, R. Sahoo, A. Roy, T. Pal, A facile synthesis of 1D nano structured selenium and Au decorated nano selenium: catalysts for the clock reaction, RSC Adv. 3 (2013) 24313-24320.

[30] Y. Liu, S. Wei, W. Gao, Ag/ZnO heterostructures and their photocatalytic activity under visible light: effect of reducing medium, J. Hazard. Mater. 287 (2015) 59-68.

[31] H.T. Ratte, Bioaccumulation and toxicity of silver compounds: a review, Environ. Toxicol. Chem. 18 (2010) 89-108.

[32] H. Wei, J. Ren, B. Han, Xu. Li, L. Han, L. Jia, Stability of polydopamine and poly (DOPA) melanin-like films on the surface of polymer membranes under strongly acidic and alkaline conditions, Colloid. Surf. B 110 (2013) 22-28.

[33] T.S. Peretyazhko, Q. Zhang, V.L. Colvin, Size-controlled dissolution of silver nanoparticles at neutral and acidic $\mathrm{pH}$ conditions: kinetics and size changes, Environ. Sci. Technol. 48 (20) (2014) 11954-11961. 